\title{
EL FOMENTO DE LA EDUCACIÓN INTERCULTURAL EN EL AULA. IMPLEMENTACIÓN DEL PROYECTO EUROSUR EN ARAGÓN
}

\author{
Antonio Gutiérrez Resa \\ Luis Ángel Puzo*
}

Implementación del proyecto EUROSUR en Aragón.

El fenómeno de la educación intercultural en el aula

Los estudiantes de enseñanza secundaria en Aragón

Las fases de realización del proyecto EUROSUR en Aragón

Conocimiento/desconocimiento de los conceptos utilizados

Elección de modelos y tipo de enseñanza recibida

Resultados. Grado de conocimiento postest

Conclusiones y acciones positivas realizadas

Referencias Bibliográficas

\section{IMPLEMENTACIÓN DEL PROYECTO EUROSUR EN ARAGÓN}

Lo que presentamos es el resultado de un proyecto financiado por el Ministerio de Educación y Cultura (Dirección General de Enseñanza Superior e Investigación Científica) dentro del III Plan Nacional de Investigación Científica y Desarrollo Tecnológico (Programa Sectorial de Promoción General del Conocimiento) durante el año 1998-99.

Este informe corresponde a la implementación del modelo EUROSUR en Aragón, modelo diseñado por un equipo de investigadores ${ }^{1}$ del Departamento de Trabajo Social y Servicios Sociales de la Escuela Universitaria de Trabajo Social de la Universidad de Valencia. Puesto a prueba, el modelo, a través de la

\footnotetext{
* Antonio Gutiérrez Resa es Catedrático de Servicios Sociales y Trabajo Social en la Escuela Universitaria de Estudios Sociales de la Universidad de Zaragoza y Luis Angel Puzo es becario y Diplomado en Trabajo Social.
} 
experimentación, se llega a obtener resultados satisfactorios. La hipótesis principal es que el factor conocimiento (información a través de tres vías: audiovisual, escrita $\mathrm{y}$ oral) es una variable independiente determinante para atenuar e incluso modificar en los jóvenes la configuración de prejuicios racistas ante los inmigrantes.

Este proyecto (modelo EUROSUR) se llegó a realizar en la Comunidad Valenciana en el año 1.996, y los resultados están publicados en la revista: Cuadernos de Trabajo Social, ${ }^{\circ} 9$ (pp. 97-107).

Junto con la implementación en Aragón, en el periodo Enero- Abril de 1.999, se ha realizado en los territorios de Castilla-La Mancha, Andalucía Occidental y Valencia, dentro de un proyecto estatal subvencionado por el Ministerio de Trabajo y Asuntos Sociales ${ }^{2}$.

El fomento de la educación intercultural en el aula

\section{Objetivos Generales:}

- Que el modelo EUROSUR, puesto a prueba a través de experimentación, obtiene resultados satisfactorios.

- Fortalecer la red de Servicios Sociales, con el propósito de promover actitudes que favorezcan la convivencia multicultural.

\section{Objetivos Específicos:}

- Analizar el grado de conocimiento de los estudiantes que están cursando enseñanza secundaría en relación a una serie de conceptos relacionados con el fenómeno de la inmigración.

- Obtener resultados y contrastarlos con los de las otras comunidades que participan en el proyecto, para obtener una visión más global (representativa).

\section{LOS ESTUDIANTES DE ENSEÑANZA SECUNDARIA EN ARAGÓN}

El proyecto se ha desarrollado con estudiantes de enseñanza secundaria de edades comprendidas entre 15 y 17 años, pertenecientes a centros públicos y privados de la Comunidad Autónoma Aragonesa, elegidos a través de muestreo aleatorio simple. El universo muestral estaba constituido por 69.270 estudiantes de enseñanza secundaria $^{3}$ de la comunidad autónoma aragonesa. La muestra la han conformado 507 estudiantes participantes (24,3\%), 252 (49,7\%) hombres y 255 (50,3\%) mujeres; de ellos, 297 (el 58,6\%) pertenecían a centros privados y 210 (el 41,4\%) restante estudiaba en centros públicos. El total de estudiantes se distribuyó en torno al 50\% entre los grupos de intervención $(248=48,9 \%)$ y de los de no intervención $(259=51,1 \%)$. Los estudiantes pertenecían a 9 centros distintos de las tres provincias. El 27,6\% cursaba $1^{\circ}$ Bachillerato Logse, el 14,8\% $2^{\circ}$ BUP, el 11,7\% $3^{\circ}$ BUP, el $13,2 \% 3^{\circ} \mathrm{ESO}$, el $19,5 \% 4^{\circ} \mathrm{ESO}$ y el $13,2 \%$ COU. 
La Comunidad Autónoma Aragonesa ${ }^{4}$, según el censo de 1.995, tenía 1.205.663 habitantes (un 3\% de la población española).

Aragón es, tras Castilla-La Mancha, la comunidad española de menor densidad poblacional, con $25 \mathrm{~h} / \mathrm{km} 2$.

Las provincias de Huesca y Teruel son las más vacías, con 13 y 10 h/km2 respectivamente. Zaragoza tiene $49 \mathrm{~h} / \mathrm{km} 2$.

La superficie es de $47.500 \mathrm{~km} 2$, superior a países como Dinamarca, Holanda o Suiza.

Aragón es nexo natural entre dos de los ejes de desarrollo más importantes de Europa: el eje atlántico y el eje mediterráneo.

\section{LAS FASES DE REALIZACIÓN DELPROYECTO EUROSUR EN ARAGÓN}

Al tratarse de un estudio piloto y preliminar se decidió, para comprobar las hipótesis de partida, aplicar un diseño cuasi experimental. Este tipo de diseño comporta una serie de desventajas, tales como la imposibilidad de llevar a cabo un control experimental estricto ya que la variable independiente no se manipula directamente por el experimentador, sino que se selecciona y cuando actúa se observan los cambios producidos en la variable dependiente. Estos diseños, no siendo tan válidos como los estrictamente experimentales, ayudan a acotar las posibles explicaciones a los fenómenos sociales, rechazando las hipótesis alternativas y afinando en la futura explicación casual de tales fenómenos. De entre los diseños cuasi-experimentales, se ha elegido el diseño temporal con pre-test y pos-test, por medio del cual podríamos obtener observaciones antes y después de la aplicación de la variable independiente a lo largo de un período determinado.

El desarrollo del programa implica las siguientes fases (Véase cuadro 1):

$11^{\mathrm{a}}$ Fase:

La implementación del programa EUROSUR comienza con una primera fase en la que se selecciona la muestra de jóvenes que cursan estudios en el segundo grado de enseñanza secundaria obligatoria (ESO) o en PUB. Si bien la muestra es al azar, se parte de un universo de centros de enseñanzas públicas y otro de enseñanzas privadas.

2 Fase:

Esta fase se corresponde con el primer contacto con los estudiantes.

- Primero se procedió a obtener una línea base de la información sobre los 12 conceptos relevantes vinculados al fenómeno de la inmigración y sus consecuencias sociales.

Los conceptos seleccionados son los siguientes: asilado, etnocentrismo, 
xenofobia, nacionalismo, inmigrante, grupo étnico, discriminación, refugiado, prejuicio, racismo, solidaridad y segregación. Han sido elegidos porque en ellos subyacen las representaciones sociales y culturales de la sociedad receptora.

- En segundo lugar, se entregó información escrita sobre tres formas de entender el fenómeno de la inmigración, recogidas en tres modelos, debiendo elegir cada estudiante uno de ellos:

Modelo R: modelo de asimilación.

Modelo G: modelo tolerante.

Modelo Y: modelo multicultural/antirracista.

En los tres modelos se contemplan contenidos cognitivos y comportamentales.

- En tercer lugar, se obtuvo una línea-base sobre los comportamientos solidarios y positivos realizados por los estudiantes de la muestra hasta la fecha hacia grupos de inmigrantes. Para ello se les pidió a los jóvenes que describiesen por escrito las posibles acciones positivas realizadas a favor de este grupo de población.

\section{$3^{\mathrm{a}}$ Fase:}

Una semana después se inicio la fase de intervención, únicamente con los estudiantes del grupo experimental. Durante esta fase los jóvenes recibían información a través de tres modalidades:

- Información escrita. Los estudiantes contrastan las definiciones de los conceptos elaborados por el equipo de investigación con sus propias nociones expresadas por escrito la semana anterior.

- Información audiovisual. Visualización del vídeo titulado: "Mediterránea Viva", elaborado por el propio equipo investigador, que contiene información acerca de elementos comunes a diferentes culturas próximas al Mediterráneo que se nos muestran aparentemente diferentes.

- Información Oral. Pretende incorporar una información directa protagonizada y expresada por las propias asociaciones de inmigrantes en nuestro país, que transmitirán su cultura autóctona y su programa de acción a los estudiantes en el aula.

\section{Fase:}

Transcurridos tres meses se procede a la fase de contrastación: Postest. En esta fase los estudiantes, tanto del Grupo Experimental como del Grupo Control, eligen de nuevo los modelos (R, G, Y) y se comprueban asimismo los comportamientos solidarios y positivos desde la intervención en el aula (se recogen las posibles acciones positivas que hayan realizado los estudiantes durante ese período de tiempo). 
CUADRO 1. FASES DEL PROGRAMA EUROSUR.

FASES

F.1. Selección muestra

F.2. Primer pase:

Testar conocimientos (grupos control

y experimental)

Elección modelos (grupos control y

experimental)

Línea-base acciones positivas (grupos

control y experimental)

F.3. Segundo pase:

Visualización vídeo (g. experimental)

Contrastar definiciones (g. expe.)

Charla (g. experimental)

ANÁLISIS ESTADÍSTICO DE DATOS

F.4. Postest:

Elección modelos (grupos control y

experimental)

Acciones positivas (grupos control y

experimental)

ANÁLISIS ESTADÍSTICO DE DATOS
TEMPORALIZACION

2 meses

2 meses

2 meses

1 mes

2 meses

1 mes

\section{CONOCIMIENTO/DESCONOCIMIENTO DE LOS CONCEPTOS UTILIZADOS}

\section{Grado de conocimiento entre todos los sujetos}

En este apartado se va a analizar el grado de conocimiento de los estudiantes respecto a cada uno de los conceptos considerados, tanto entre todos los jóvenes como en función del tipo de enseñanza (pública/privada).

Los conceptos que mayoritariamente desconocen los estudiantes son (Véase gráfico $\mathrm{n}^{\circ} 1$ ): segregación, asilado, etnocentrismo y prejuicio. Entre los conceptos cuyo grado de conocimiento del estudiante se encuentra por encima del $50 \%$, están los siguientes: refugiado, nacionalismo, racismo, grupo étnico, xenofobia, solidaridad, discriminación e inmigrante. 
Los valores promedio alcanzados son los siguientes:

$\begin{array}{lc}\text { CONCEPTO } & \text { PROMEDIO } \\ \text { Segregación } & 0.098 \\ \text { Asilado } & 0.128 \\ \text { Etnocentrismo } & 0.366 \\ \text { Prejuicio } & 0.406 \\ \text { Refugiado } & 0.597 \\ \text { Nacionalismo } & 0.656 \\ \text { Racismo } & 0.658 \\ \text { Grupo étnico } & 0.708 \\ \text { Xenofobia } & 0.765 \\ \text { Solidaridad } & 0.785 \\ \text { Discriminación } & 0.994 \\ \text { Inmigrante } & 1.003\end{array}$

Se puede adelantar la conclusión siguiente: que la mayoría de los participantes tiene un escaso conocimiento de los doce conceptos seleccionados. No obstante tras la intervención, en general se obtienen unos porcentajes de conocimiento más elevados.

Gráfico 1: Grado de conocimiento

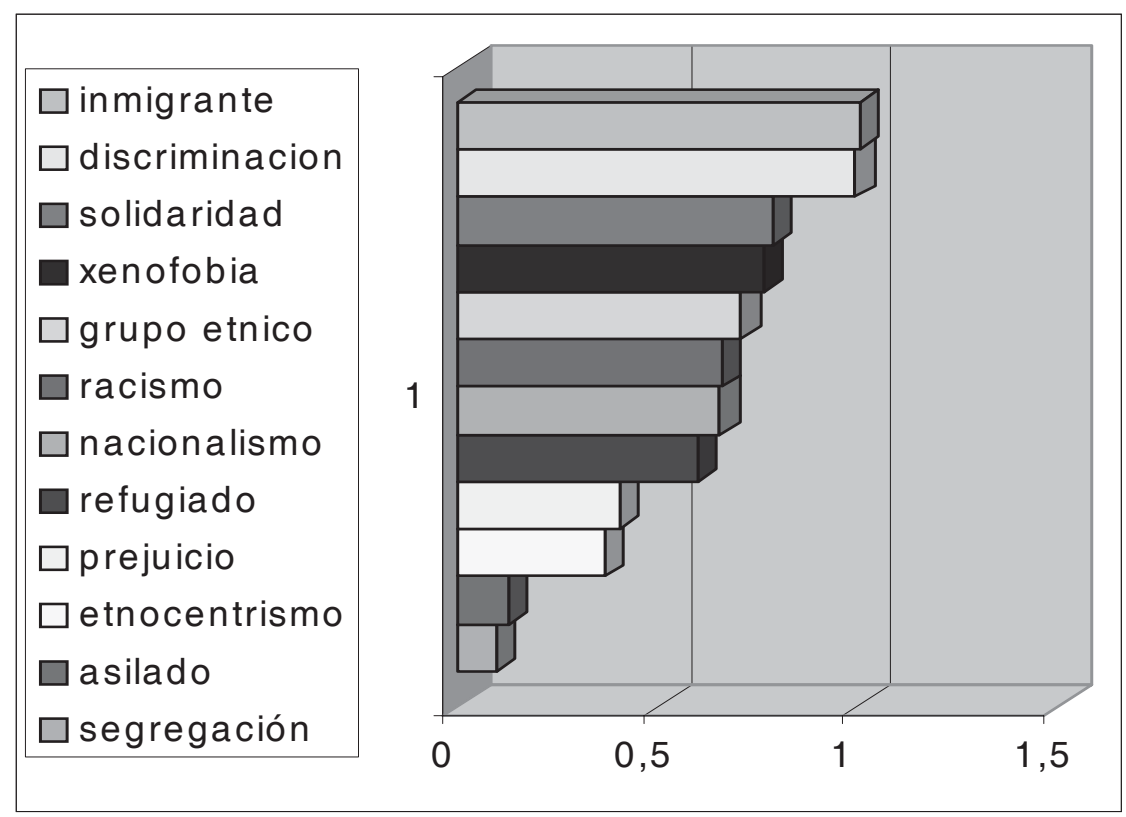


La mayoría de los estudiantes, indistintamente del tipo de educación recibida, tiene un escaso conocimiento de los doce conceptos. Se puede comprobar que los conceptos más desconocidos son: segregación (90.3\%), asilado (87.4\%), etnocentrismo (67.1\%) y prejuicio (59.8). En cambio aquéllos que tienen un mayor conocimiento son: inmigrante (98.6\%), discriminación (97.2\%) y solidario (78.3\%).

En el estudio realizado en Valencia ${ }^{5}$ se obtienen resultados muy similares. Coinciden los grupos de los conceptos menos conocidos y los que superan el $50 \%$ del grado de conocimiento. Pero con alguna variación dentro del orden de cada grupo.

\section{GRADO DE CONOCIMIENTO Y TIPO DE CENTRO}

Respecto del tipo de enseñanza recibida (colegio público/privado), no existen grandes diferencias en el grado de conocimiento de los distintos conceptos.

Grado de conocimiento y tipo de centro

$\begin{array}{llll}\mathrm{N}^{\circ} & \text { CONCEPTO } & \text { PÚBLICO } & \text { PRIVADO } \\ \text { GRÁFICO } & & \end{array}$

$\begin{array}{lllll}1 & \text { asilado } & 0,147 & 0,114 & 0,128 \\ 2 & \text { discriminacion } & 1,004 & 0,986 & 0,994 \\ 3 & \text { etnocentrismo } & 0,342 & 0,383 & 0,336 \\ 4 & \text { refugiado } & 0,671 & 0,545 & 0,597 \\ 5 & \text { xenofobia } & 0,761 & 0,767 & 0,765 \\ 6 & \text { prejuicio } & 0,409 & 0,404 & 0,406 \\ 7 & \text { nacionalismo } & 0,68 & 0,639 & 0,656 \\ 8 & \text { racismo } & 0,609 & 0,693 & 0,658 \\ 9 & \text { inmigrante } & 1,019 & 0,993 & 1,003 \\ 10 & \text { solidaridad } & 0,804 & 0,771 & 0,785 \\ 11 & \text { grupo étnico } & 0,676 & 0,73 & 0,708 \\ 12 & \text { segregación } & 0,071 & 0,117 & 0,098\end{array}$

Puede observarse que los participantes de la enseñanza privada tienen un conocimiento muy similar al que poseen los de la enseñanza pública. 
Gráfico 2: Grado de conocimiento (público-privado).

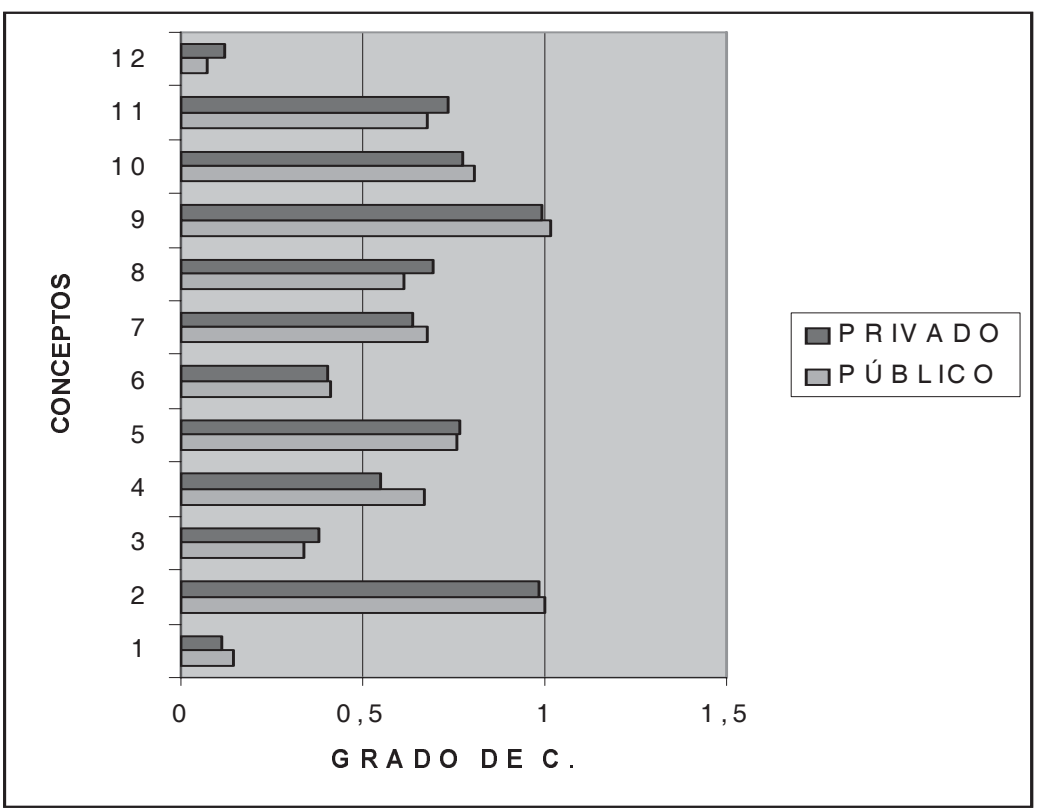

En el estudio realizado en Valencia se obtienen resultados muy similares.

\section{ELECCIÓN DE MODELOS Y TIPO DE ENSEÑANZA RECIBIDA}

\section{Elección de modelos}

En este apartado analizaremos si el tipo de educación recibida (pública/ privada) o el efecto de la intervención realizada tiene una influencia sobre la elección de un modelo tolerante (modelo G), antirracista (modelo Y) o racista (modelo R). Tomando a todos los sujetos que forman parte del trabajo, con los datos obtenidos en el pretest, el 26,13\% elige el modelo G, el 55,24\% el modelo Y, y el 18,61\% el modelo R. En el postest, el 25,94\% elige el modelo G, el 53,86\% el modelo Y, y el 20,19\% el modelo R.

Estos datos a nivel descriptivo tienen una lectura distinta cuando se analizan en función del tipo de educación recibida y el grupo de intervención/no intervención.

\section{Elección de modelos y tipo de enseñanza recibida}

Respecto al tipo de educación recibida, si que aparecen diferencias estadísticamente significativas. En el pretest, el 33,86\% de los estudiantes de 
enseñanza privada eligen el modelo $\mathrm{Y}$ (antirracista), frente al 21,39\% de los estudiantes de enseñanza pública. El 15,84\% de los estudiantes de enseñanza privada eligen el modelo $\mathrm{G}$ (tolerante), frente al 10,30\% de los estudiantes de enseñanza pública. En el modelo R (racista) no se aprecian diferencias significativas, 9,70\% pública, frente al $8,91 \%$ privada (Gráfico 3).

Gráfico 3: Elección de modelo y tipo de enseñanza recibida.

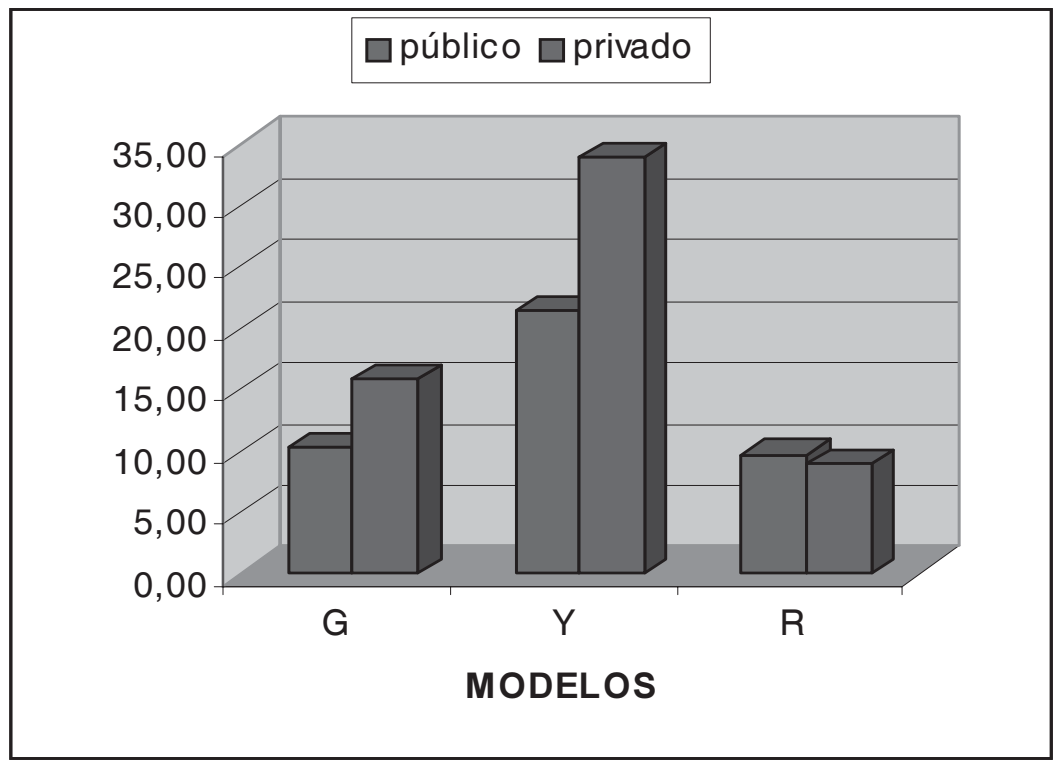

Estos resultados indican que los estudiantes que reciben una educación privada eligen en mayor medida modelos antirracistas, en el pretest, frente a los estudiantes de enseñanza pública.

Los datos presentados contrastan con los obtenidos en el estudio de Valencia, que son opuestos a los de Aragón.

\section{RESULTADOS. GRADO DE CONOCIMIENTO POSTEST}

\section{Grado de conocimiento entre sujetos del grupo intervención/no intervención}

En este apartado se va a analizar el grado de conocimiento de los estudiantes respecto a los conceptos y su pertenencia al grupo de intervención/no intervención.

Los resultados son claros. Los estudiantes del grupo intervención (experimental) mejoran respecto al grupo no intervención (control). 
Grado de conocimiento y tipo de grupo

$\begin{array}{llcc}\mathrm{N}^{\circ} \text { GRÁFICO } & \text { CONCEPTO } & \text { CONTROL } & \text { EXPERIMENTAL } \\ 1 & \text { asilado } & 0,265 & 0,32 \\ 2 & \text { discriminación } & 0,96 & 0,967 \\ 3 & \text { etnocentrismo } & 0,359 & 0,514 \\ 4 & \text { refugiado } & 0,718 & 0,791 \\ 5 & \text { xenofobia } & 0,795 & 0,809 \\ 6 & \text { prejuicio } & 0,381 & 0,442 \\ 7 & \text { nacionalismo } & 0,618 & 0,776 \\ 8 & \text { racismo } & 0,298 & 0,51 \\ 9 & \text { inmigrante } & 0,977 & 0,989 \\ 10 & \text { solidaridad } & 0,386 & 0,338 \\ 11 & \text { grupo étnico } & 0,602 & 0,773 \\ 12 & \text { segregación } & 0,055 & 0,143\end{array}$

Se puede apreciar lo que decíamos más arriba. : que tras la intervención el grupo experimental mejora respecto del grupo de control.

Gráfico 4: Grado de conocimiento y grupo

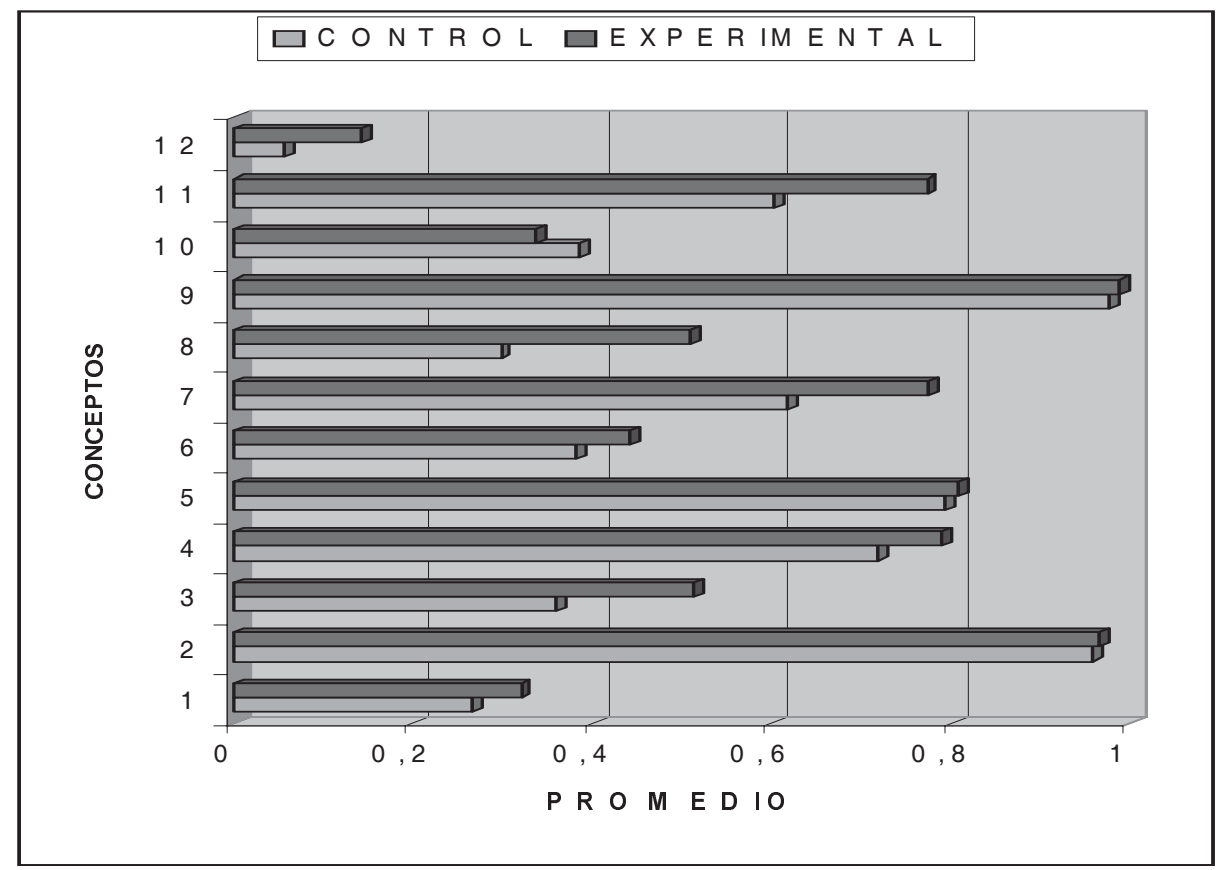




\section{ELECCIÓN DE MODELOS}

En este apartado se va a analizar el modelo elegido por los estudiantes de los grupos intervención (experimental) y no intervención (control).

Para analizar mejor los datos se incluyen dos gráficos y sus tablas respectivas, que corresponden al pretest $\mathrm{y}$ al postest.

Elección de modelo en el pretest por grupo de intervención y no intervención.

$\begin{array}{lll}\text { PRETEST } & \text { CONTROL } & \text { EXPERIMENTAL } \\ \text { G } & 29,06 & 23,07 \\ \text { Y } & 51,16 & 59,51 \\ \text { R } & 19,76 & 17,4\end{array}$

Gráfico 5: Elección del modelo en el pretest.
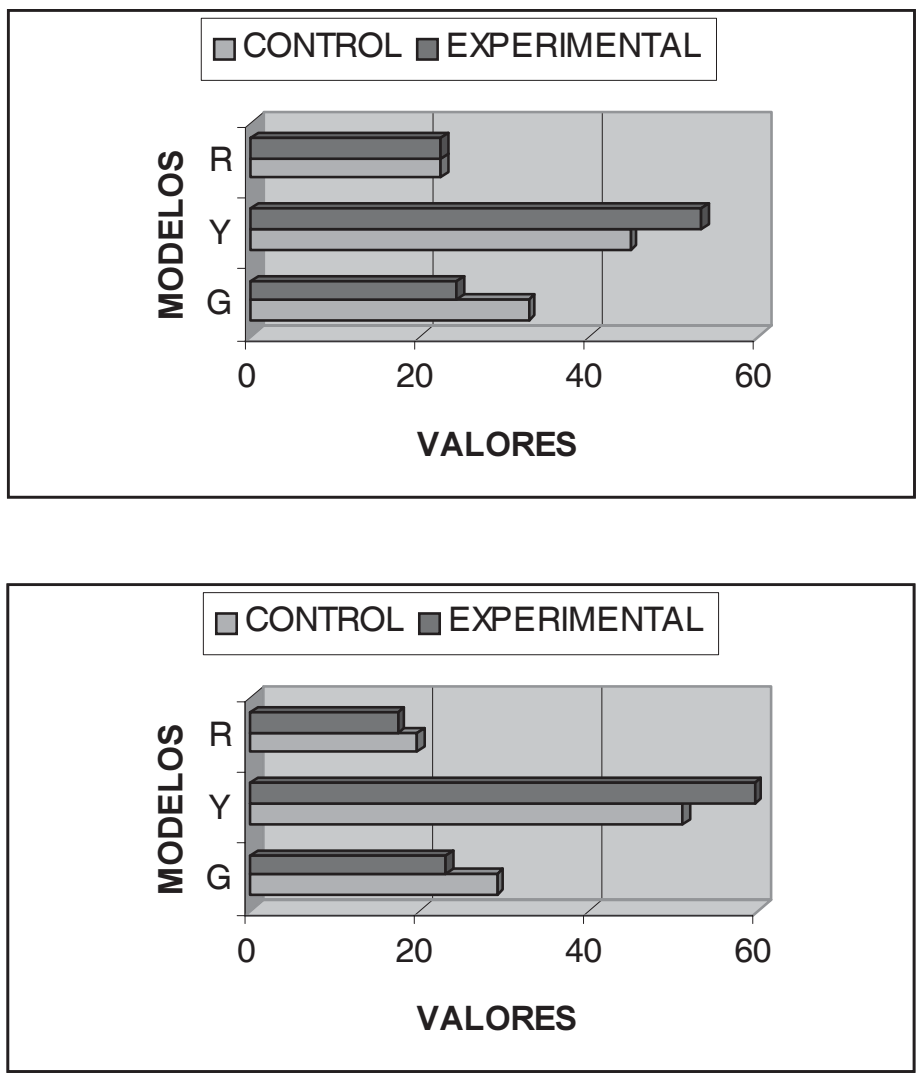
Elección de modelo en el postest por grupo de intervención y no intervención.

$\begin{array}{lll}\text { POSTEST } & \text { CONTROL } & \text { EXPERIMENTAL } \\ \text { G } & 32,78 & 24,29 \\ \text { Y } & 44,81 & 53,27 \\ \text { R } & 22,4 & 22,42\end{array}$

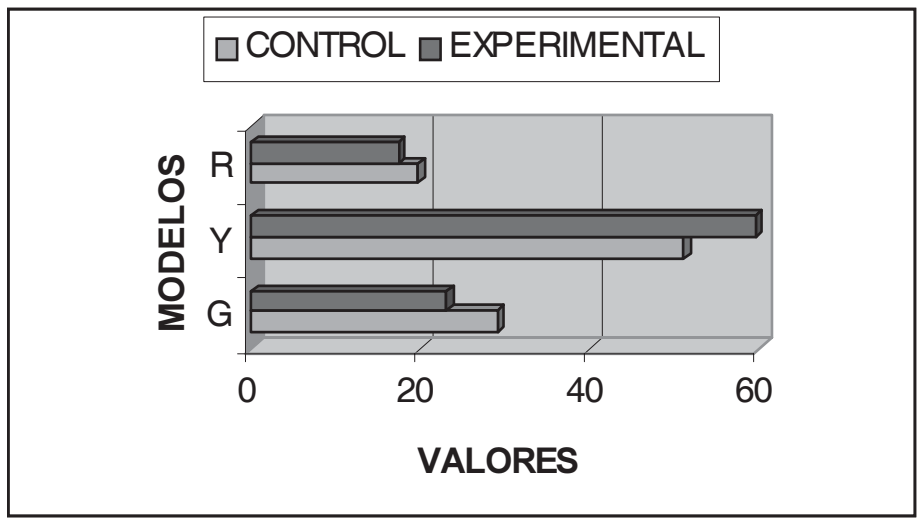

\section{CONCLUSIONES Y ACCIONES POSITIVAS REALIZADAS}

\section{Conclusiones:}

-El grupo de intervención (experimental) se mantiene en el pretest y el postest, mayoritario en la elección del modelo antirracista (modelo Y).

-El grupo de no intervención consolida en el postest posiciones menos tolerantes, con la disminución en la elección del modelo antirracista (modelo Y) y el aumento del modelo racista (modelo $\mathrm{R}$ ).

-Las mujeres eligen de forma significativa modelos más tolerantes. Además la intervención acentúa aún más estos resultados.

-Tanto en el pretest como en el postest, existe un desconocimiento de los conceptos analizados. Cuando se relaciona el grado de conocimiento y el tipo de enseñanza recibida, existe un mayor porcentaje de casos en la enseñanza privada que tienen algún grado de conocimiento, frente a la enseñanza pública, de los conceptos.

\section{ACCIONES POSITIVAS REALIZADAS}

Aunque se han contado más acciones en el pretest, las realizadas en el postest tienen un valor, cualitativamente, más representativo, debido a la desigualdad temporal que abarca cada uno de los dos momentos. 
Las acciones realizadas en el pretest (débiles y fuertes) son cuantitativamente casi las mismas en el grupo experimental (45) y en el grupo control (37). Por tipo de centro (público/privado) hay un reparto al $50 \%$.

En el postest se incrementan el número de acciones (fuertes y débiles) en los centros privados ( 34 en los privados, frente a 22 en los públicos). Es el resultado de la participación de sus alumnos en actividades (propuestas por el profesorado) relacionadas con el tema del proyecto y su mayor presencia en O.N.G's de ámbito religioso.

Tendríamos que analizar los datos más en profundidad para confirmar si los estudiantes de centros privados han estado condicionados, por las actividades que realizan, en la realización de mayor número de acciones en el postest. 


\section{NOTAS}

${ }^{1}$ Jordi GARCÉS F.

Francisco RÓDENAS R.

Susana SÁNCHEZ F.

Inmaculada VERDEGUER A.

${ }^{2}$ En la Comunidad Autónoma Aragonesa la investigación la realizaban Antonio Gutiérrez Resa y Luis Angel Puzo Mur

${ }^{3}$ Fuente: Informe anual del Consejo Económico y Social de Aragón 1997. Datos curso 96-97.

${ }^{4}$ Fuente: página web del Gobierno de Aragón (http://www.aragob.es/acerca.htm).

${ }^{5}$ Informe del proyecto: Activación de actitudes interculturales en el medio educativo.

Cit. Bibliografía.

\section{BIBLIOGRAFÍA}

COLECTIVO IOE., (1993): La inmigración (conversaciones 92), Madrid, Popular. GARCÉS, I., RÓDENAS, F., SÁNCHEZ, S. y VERDEGUER, I., "Inmigrantes en España. Heurísticos para la interculturalidad", en GARCÉS FERRER, J. y MARTÍNEZ ROMÁN, Ma A., (cord.) (1996): Bienestar social y necesidades especiales, Tiran lo Blanch, Valencia.

GARCÉS, I. , RÓDENAS, F., SÁNCHEZ, S., VERDEGUER, I., "La información como potenciador de la convivencia intercultural", en HERNÁNDEZ SACRISTÁN, C. y MORANT MARCO, R., (eds.), (1997): Lenguaje y Emigración, Universitat de València, Valencia.

GARCÉS, I., RÓDENAS, F., SÁNCHEZ, S., VERDEGUER, I.: "Trabajo Social con poblaciones receptoras de inmigrantes: Un modelo cuasi experimental para fomentar actitudes multiculturales", en Cuadernos de Trabajo Social, 9, (1996): 97-107.

GARCÉS, I., RÓDENAS, F. y Do ROSARIO, Ma: "Investigaçao transcultural sobre atitudes face aos imigrantes: Estudo piloto de Lisboa", en SociologíaProblemas e Práticas, 25, Lisboa, (1997): 139-153.

GARCÉS, I, RÓDENAS, F., SÁNCHEZ, S., VERDEGUER, I. y Do ROSARIO, $M^{a}$ : "Trabalho social com populaçoes receptoras de emigrantes: Um modelo quase experimental para o fomento de atitudes multiculturais", en Psicologia: Teoria, Investigaçao e Prática, vol. 2, 1, Lisboa, (1997): 73-84.

VV.AA., (1997): La educación en Aragón, en Informe Anual del Consejo Económico y Social de Aragón, Zaragoza, DGA.

VV.AA., (1998): Observatorio permanente de la inmigración, Ministerio de Trabajo y Asuntos Sociales, Madrid. 